Heming Huang, Kevin Schires, Frédéric Grillot, Dejan Arsenijević, Tagir Sadeev, Dieter Bimberg

\title{
InAs/GaAs excited state quantum-dot transmitters operating under long-delayed optical feedback
}

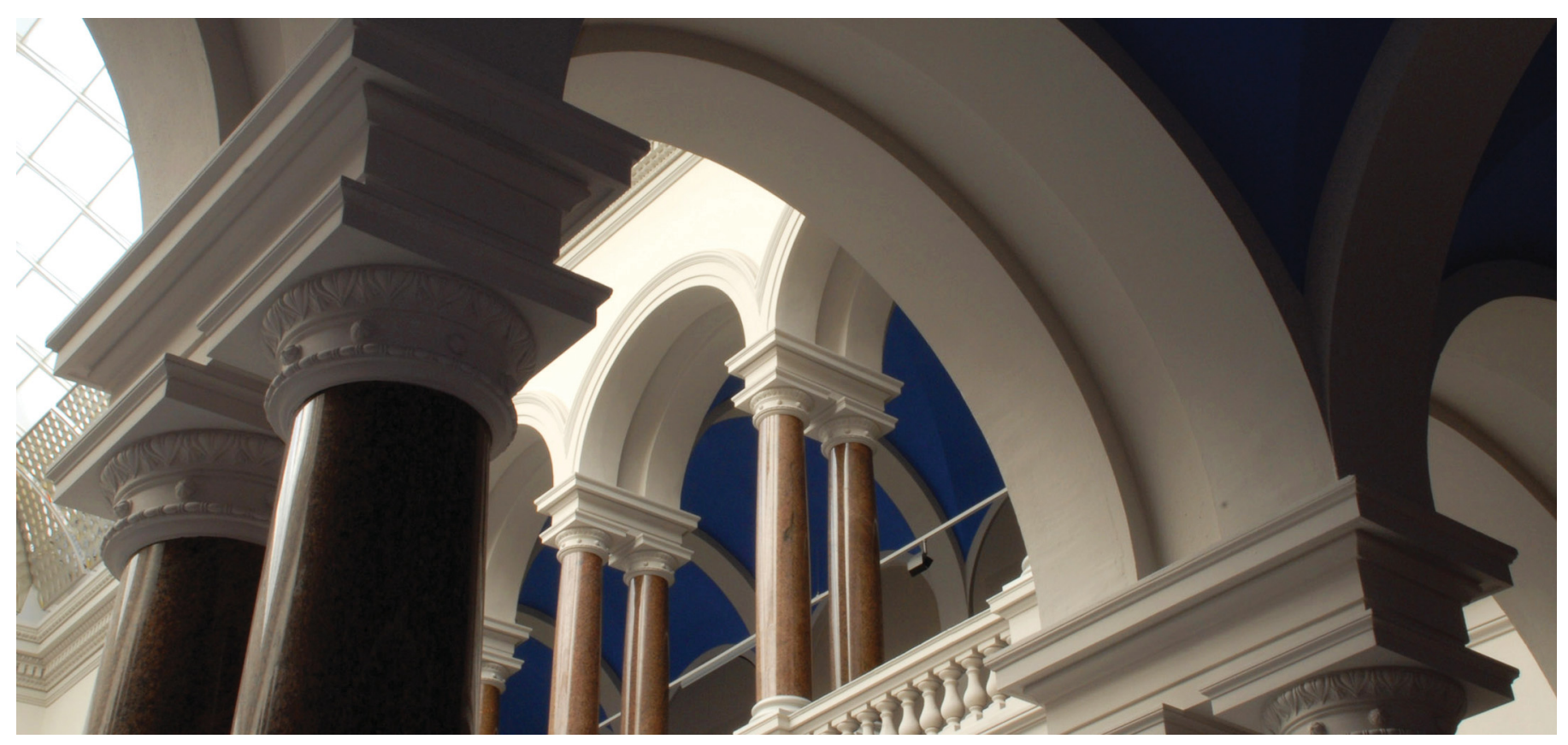

Huang, H., Schires, K., Grillot, F., Arsenijević, D., Sadeev, T., \& Bimberg, D. (2016). InAs/GaAs excited state quantum-dot transmitters operating under long-delayed optical feedback. Proc. SPIE 9892, Semiconductor Lasers and Laser Dynamics VII, 98920C (28 April 2016). https://doi.org/10.1117/12.2227953. 


\title{
InAs/GaAs excited state quantum-dot transmitters operating under long-delayed optical feedback
}

\author{
Heming Huang ${ }^{a^{*}}$, Kevin Schires ${ }^{\mathrm{a}}$, and Frédéric Grillot ${ }^{\mathrm{a}, \mathrm{b}}$ \\ anniversité Paris-Saclay, Télécom ParisTech, CNRS LTCI, 46 rue Barrault, 75013 Paris, France \\ ${ }^{\mathrm{b}}$ Center for High Technology Materials, University of New-Mexico, 1313 Goddard SE, \\ Albuquerque, NM, USA \\ Dejan Arsenijevićc ${ }^{c}$ Tagir Sadeev ${ }^{\mathrm{c}}$, Dieter Bimberg ${ }^{\mathrm{c}, \mathrm{d}}$, \\ 'Institut für Festkörperphysik, Technische Universität Berlin, 10623 Berlin, Germany \\ ${ }^{\mathrm{d}}$ Faculty of Engineering, King Abdulaziz University, Jeddah 21589, KSA \\ *heming.huang@telecom-paristech.fr
}

\begin{abstract}
The sensitivity to external optical feedback of two different InAs/GaAs QD Fabry-Perot (FP) lasers with long cavities is investigated. One laser emits on the ground state while the other one emits solely on the excited state. Our results indicate that for the same bias level, the ES laser shows a larger sensitivity to external feedback, the critical level being under $1 \%$ versus above $9 \%$ for the GS laser. The ES laser exhibits an enhanced route to chaos, such that the first destabilization occurs for a lower feedback strength than for the GS laser.
\end{abstract}

Keywords: semiconductor laser, quantum dot, Fabry-Perot, optical feedback

\section{INTRODUCTION}

Quantum-dot (QD) lasers exhibit a large gain saturation factor and highly damped relaxation oscillations making them less sensitive to optical feedback as compared to quantum-well $(\mathrm{QW})$ devices ${ }^{1,2}$. QD lasers are usually engineered to operate on the ground-state (GS) because of its ultralow operation current in contrast to the excited states ${ }^{3,4}$. Several limitations exist however for QD lasers operating on the GS such as lower gain and differential gain as well as a larger gain compression factor. In addition, GS lasers do not necessarily exhibit a small phase-amplitude coupling factor, which is a drawback for chirpless operation in fiber-based optical telecommunications ${ }^{5,6}$. Thus exploiting the stimulated emission from the first ES will improve the dynamics of QD lasers, owing to twofold faster rate of carrier capture from the surrounding carrier reservoir as well as a higher saturated gain ${ }^{7,8}$. Emission from the ES can be achieved in multiple ways, one of them relying on using a dichroic mirror on the facet to fully inhibit the GS transition ${ }^{5,8}$. As a consequence, large-signal modulation up to $25 \mathrm{Gbps}$ has been recently demonstrated for an InAs/GaAs QD laser emitting on the ES transition $^{8}$. In addition, it was pointed out that ES lasers exhibit a smaller phase-amplitude coupling factor ${ }^{9,10}$ strongly influencing the nonlinear dynamic properties of ES QD lasers.

\section{DEVICE DESCRIPTION}

Two types of InAs/GaAs QD FP lasers emitting on the GS and exclusively on the ES are studied in this work. The active regions of both devices are "dots-in-a-well" structures, with ten InAs QD layers grown by molecular beam epitaxy (MBE) embedded in InGaAs $\mathrm{QW}^{10}$. For the GS laser, the cavity is $1.5 \mathrm{~mm}$ long with a $2 \mu \mathrm{m}$-wide waveguide, the threshold current is $30 \mathrm{~mA}$ at $20{ }^{\circ} \mathrm{C}$ with a wall plug efficiency of $16 \%$, and the center wavelength is around $1305 \mathrm{~nm}$. The LIV curves are presented in Figure 1(a) and the corresponding optical spectrum taken at $2 \times \mathrm{I}_{\text {th }}$ is depicted in Figure 1(b). For the ES laser, the cavity is $1 \mathrm{~mm}$ long with a $2 \mu \mathrm{m}$-wide waveguide, and the threshold current at $20{ }^{\circ} \mathrm{C}$ is $88.5 \mathrm{~mA}$ with a wall plug efficiency of $11 \%$, and the center wavelength is around $1231 \mathrm{~nm}$. The LIV curves are shown in Figure 1(c) and the corresponding optical spectrum measured at $2 \times \mathrm{I}_{\mathrm{th}}$ is depicted in Figure 1(d). Although the ES laser has a smaller cavity length, which is favorable to inhibit the GS transition, the occurrence of the sole ES can also be due to the presence of smaller size nanostructures presenting an optical transition that overlaps the ES transition peak. 

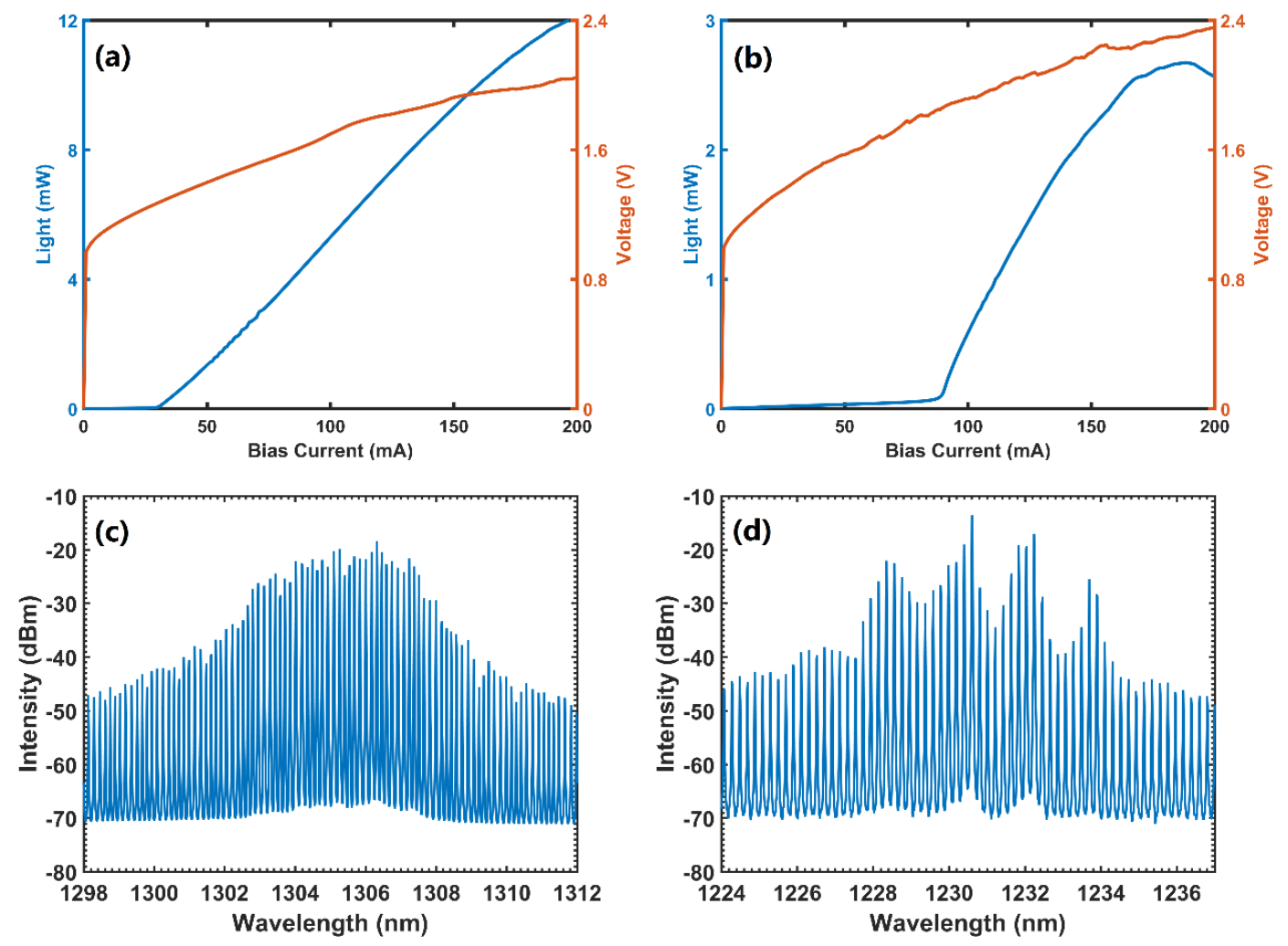

Figure 1. (a) LIV curves and (c) optical spectrum measured at $2 \times \mathrm{I}_{\text {th }}$ of the GS laser; (b) LIV curves and (d) optical spectrum measured at $2 \times \mathrm{I}_{\mathrm{th}}$ of the ES laser. All measurements are performed at $20^{\circ} \mathrm{C}$.

The QD lasers are inserted into an external optical feedback loop shown in Figure 2. The laser emission is coupled in using a lens-ended fiber, and split by a 90/10 coupler into feedback and detection paths. In the feedback path, the light is reflected back to the QD using a back-reflector (BKR), consisting of a variable attenuator and a mirror. A polarization controller in the feedback path allows matching the polarizations of the emitted and reflected light in order to balance the perturbation from the fiber birefringence and maximize the effects of the optical feedback. The length of the external cavity is around $7 \mathrm{~m}$, which corresponds to the long external cavity condition. Due to the different output divergence, the attainable feedback strength $\left(\mathrm{r}_{\mathrm{ext}}\right)$ variation range is not exactly the same for the GS and ES devices. For the GS laser, $\mathrm{r}_{\mathrm{ext}}$ is varied from $0 \%$ to $12.5 \%$, and from $0 \%$ to $5.6 \%$ for the ES one. In the detection path, isolated from the rest of the setup, electrical and optical spectrum analyzers (ESA and OSA) are used to study the feedback dynamics.

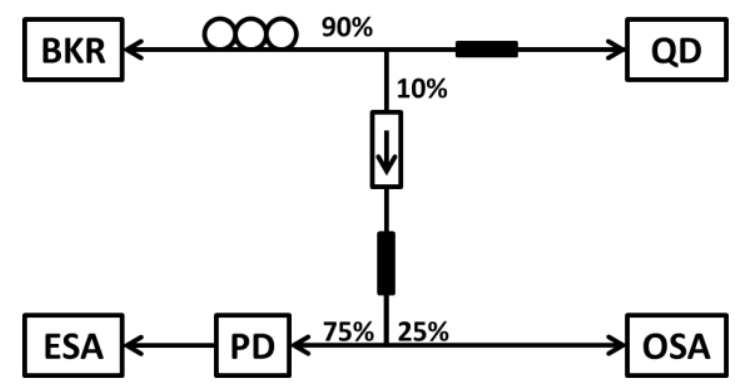

Figure 2. Schematic of the external optical feedback experimental setup. QD: Quantum-Dot device, PD: Photo detector, ESA: Electrical Spectrum Analyzer, OSA: Optical Spectrum Analyzer, BKR: Back Reflector. 


\section{RESULTS AND DISCUSSIONS}

First the feedback dynamics of the GS laser is investigated. In order to do so, bias conditions of $1.5 \times, 2 \times$ and $2.5 \times \mathrm{I}_{\mathrm{th}}$ are considered. Because the external cavity length is several meters long, the impact of the phase of the reflected wave can be neglected. Figure 3 represents the different optical and electrical spectra mapped as a function of the optical feedback strength $r_{\text {ext }}$.
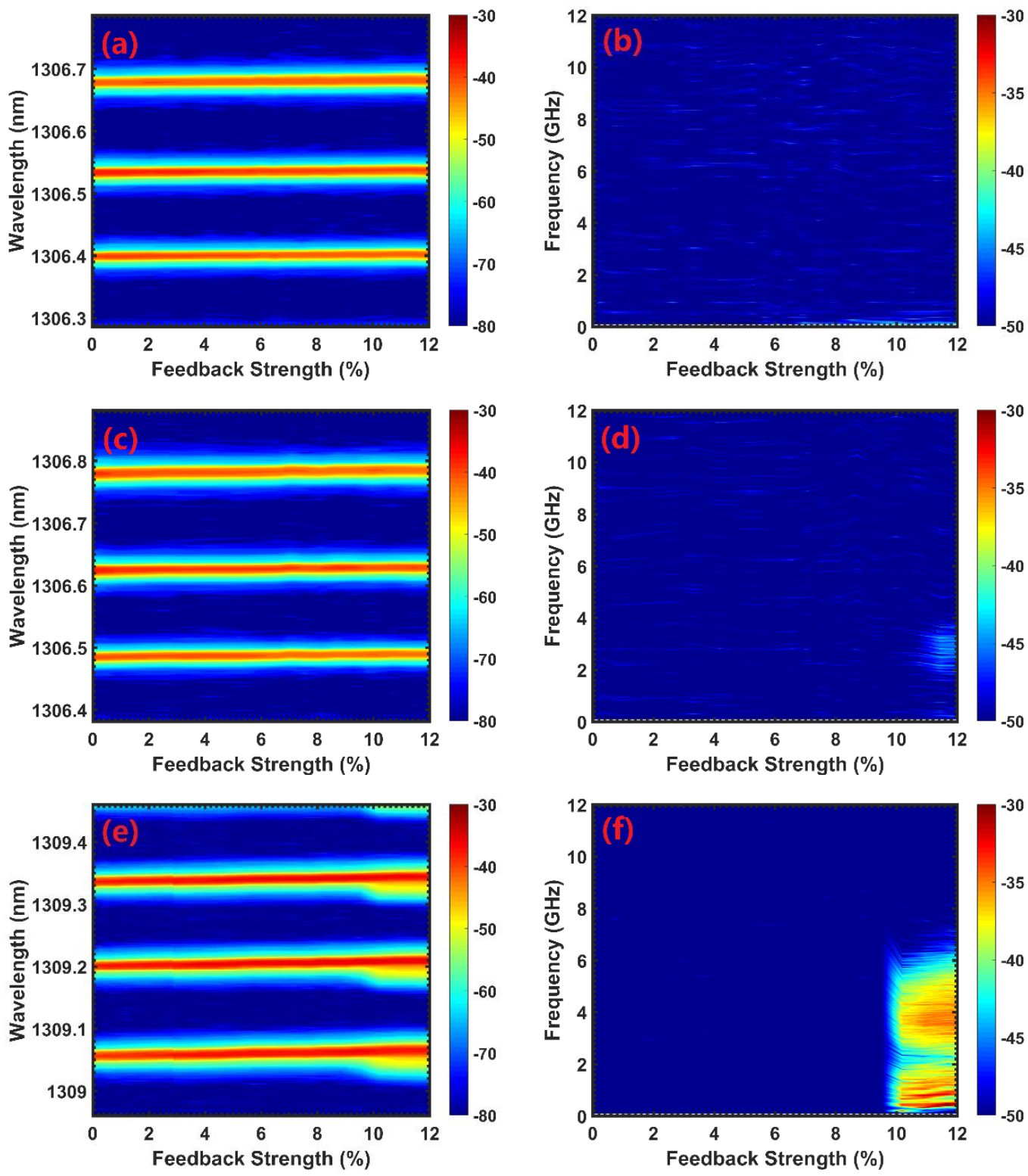

Figure 3. GS QD laser: optical spectra (a) and electrical spectra (b) mapped at $1.5 \times \mathrm{I}_{\mathrm{th}}$, at $2 \times \mathrm{I}_{\text {th }}(\mathrm{c}),(\mathrm{d})$ and $2.5 \times \mathrm{I}_{\text {th }}(\mathrm{e}),(\mathrm{f})$.

At $1.5 \times \mathrm{I}_{\mathrm{th}}$, the GS laser is found always stable across the whole range of optical feedback ranging from $0 \%$ to $12 \%$, the modal wavelength red-shifts continuously, without any sign of linewidth broadening, and the electrical spectrum remains flat independent of the feedback strength, no oscillations nor chaos are observed. At $2 \times \mathrm{I}_{\text {th }}$, the GS spectrum still remains unchanged, however, above $11 \%$ feedback strength, some oscillations start arising in the electrical spectrum, indicating that the laser starts to be perturbed by the external feedback. At $2.5 \times \mathrm{I}_{\text {th }}$, the critical feedback level ( $\mathrm{r}_{\text {crit }}$ ) corresponding to the first destabilization can be clearly identified. For feedback strengths above $9.5 \%$ the dynamics becomes more 
complex. In order to better illustrate the effects of the optical feedback, Figure 4 presents the optical and electrical spectra of the laser under free-running $\left(\mathrm{r}_{\mathrm{ext}}=0 \%\right)$ and maximal feedback $\left(\mathrm{r}_{\mathrm{ext}}=12.52 \%\right)$ conditions, respectively. As observed in the electrical spectrum once $r_{\text {ext }}>r_{\text {crit }}$, the longitudinal modes are significantly broadened and this signature corresponds to chaotic behavior. At this stage it is important to stress that the pump current has a strong importance on the laser dynamics. At lowest pump current, the laser remains unchanged for the whole range of optical feedback strength while at higher ones it can operate within the coherence collapse. In the latter case, the onset of the coherence collapse is found to be down-shifted with increasing current, contrary to DFB lasers, where the tendency is inverse ${ }^{2}$. At larger bias, the lasing mode is enhanced in a DFB laser, as well as inherent noise is suppressed, leading to a better stability against feedback, while in a FP laser, more modes start to lase, hence the growing modal competition can introduce dynamic instability to the FP laser.
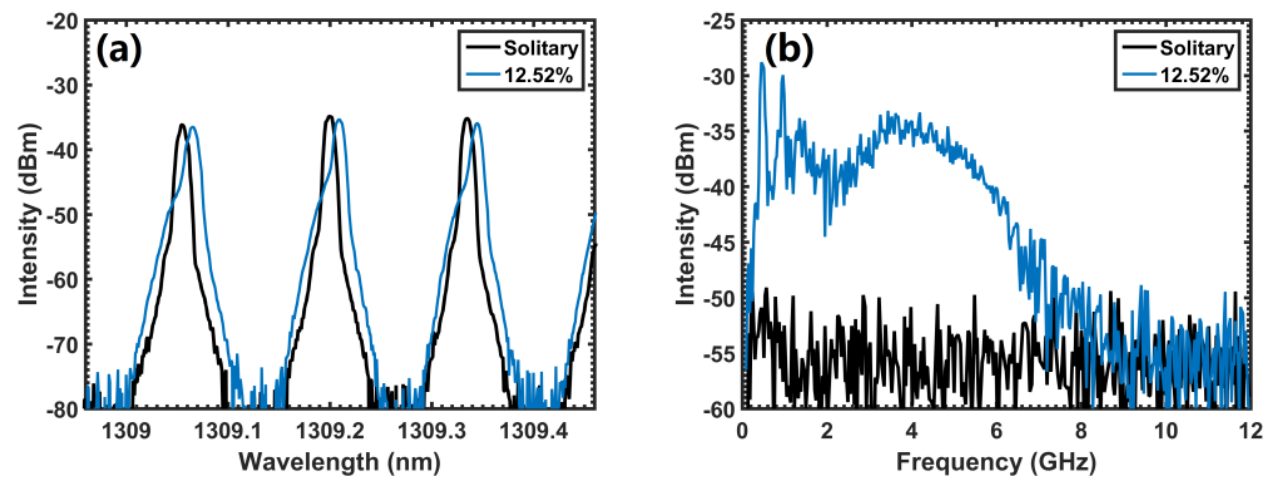

Figure 4. GS QD laser at a bias level of $2.5 \times \mathrm{I}_{\text {th: }}$ ( (a) optical and (b) electrical spectra of the free-running laser $(0 \%)$ and maximal (12.52\%) optical feedback.
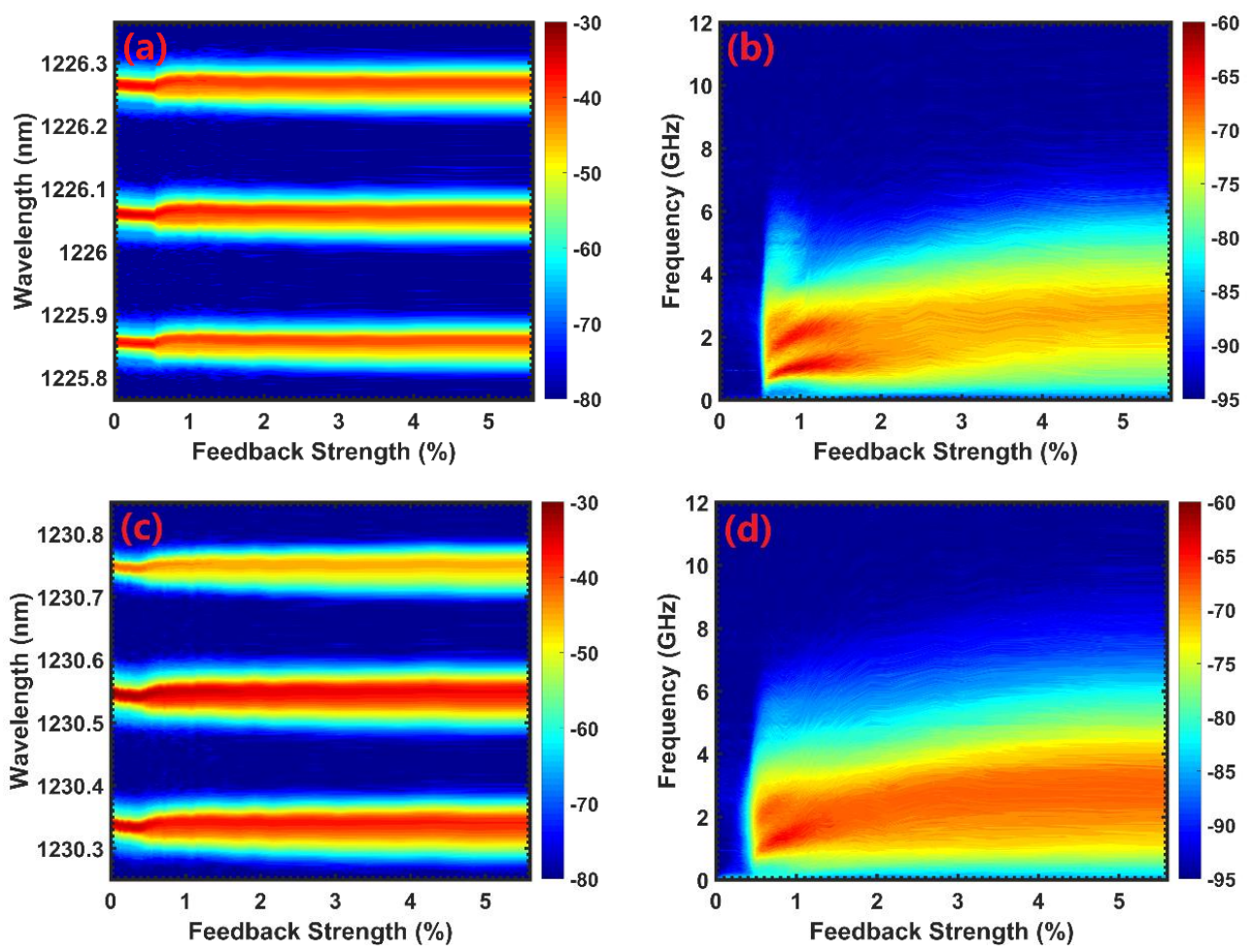

Figure 5. ES laser: optical spectra maps at (a) $1.5 \times \mathrm{I}_{\mathrm{th}}$, (c) $2 \times \mathrm{I}_{\text {th }}$, and electrical spectra maps at (b) $1.5 \times \mathrm{I}_{\mathrm{th}}$, (d) $2 \times \mathrm{I}_{\mathrm{th}}$. 
For the ES laser, the pumping conditions are fixed to $1.5 \times$ and $2 \times \mathrm{I}_{\mathrm{th}}$. Due to the rollover observed in the LI curve (Figure 1c) above $190 \mathrm{~mA}$, the pumping condition corresponding to $2.5 \times \mathrm{I}_{\mathrm{th}}(225 \mathrm{~mA})$ is not considered. Figure 5 represents the optical and electrical spectra of the ES laser measured under different currents as a function of $r_{\text {ext }}$. At $1.5 \times \mathrm{I}_{\text {th }}$, the laser remains insensitive to optical feedback till $0.5 \%$, beyond which the modes begin to broaden (Figures $5(\mathrm{a})$ and $5(\mathrm{~b})$ ). Figure 6 represents a set of optical and electrical spectra recorded at both bias levels for the ES free-running laser, and for $0.76 \%$ and $5.82 \%$ optical feedback. Figures $6(\mathrm{a})$ and $6(\mathrm{c})$ illustrate the mode broadening in the optical spectrum for both pumping levels, while figure 6(b) and 6(d) depict the destabilization in the electrical spectra. In particular, the electrical spectrum in figure $6(\mathrm{~b})$ obtained under $0.8 \%$ optical feedback shows the occurrence of quasi-periodic oscillations with high pedestal. The feedback resistance of a semiconductor laser being driven by the relaxation-oscillation damping ${ }^{13}$, the discrepancy observed in this work can be attributed to the larger damping of the GS-laser ${ }^{8}$. At higher pump currents and similar feedback strength, the peaks become less sharp and intense, as shown in Figure 6(d). Besides, the comparison between $6(\mathrm{~b})$ and $6(\mathrm{~d})$ indicates that for the same maximum feedback strength, for larger pump the chaos intensifies and the bandwidth increases. These results are in agreement with the decreasing stability of the FP laser at higher bias, as mentioned before. Finally let us emphasize that at higher pump, the laser appears even more sensitive to the optical feedback, since the critical level is found to decrease down to $0.4 \%$ as opposed to $9 \%$ for the QD laser emitting on the GS transition.
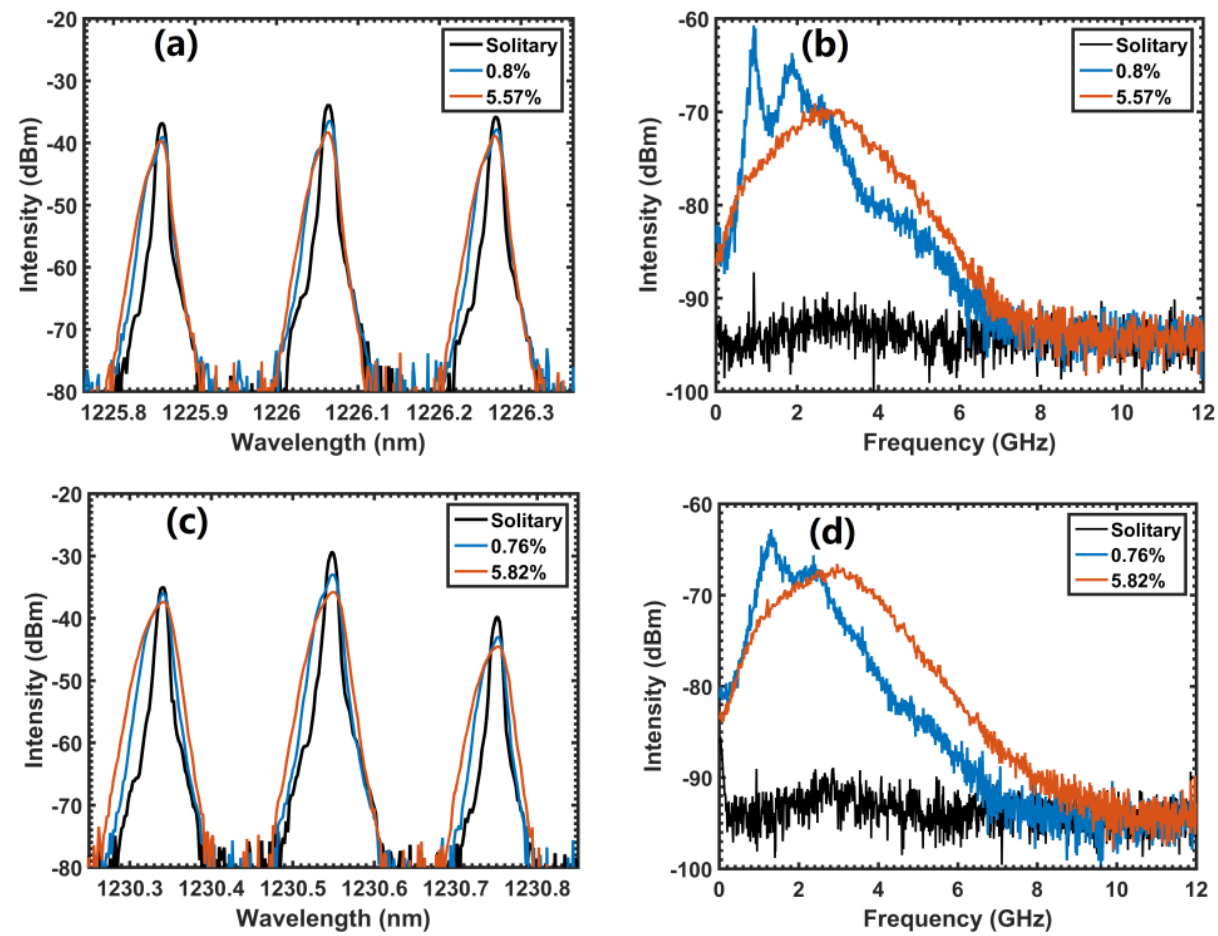

Figure 6. ES QD laser at $1.5 \times \mathrm{I}_{\text {th }}$ bias level: (a) optical and (b) electrical spectra of the free-running laser, $0.8 \%$ and $5.57 \%$ optical feedback; ES laser at $2 \times \mathrm{I}_{\text {th }}$ bias level: (c) optical and (d) electrical spectra of the free-running laser, $0.76 \%$ and $5.82 \%$ optical feedback.

Thus laser ES emission is found to be more sensitive to optical feedback, with $r_{\text {crit }}<1 \%$ for different bias levels, while for the GS laser $r_{\text {crit }}>9 \%$ for the highest bias level under study. In addition, quasi-periodic behavior is observed in the ES laser within a rather tight window of injection strengths, while this is not the case for the GS laser. The difference between the GS and ES emission can be explained in the following way: - the lower damping rate of the ES as already reported in [14], [15], - the different free spectral ranges combined with the shape of the optical spectra can also reflect the intensity of the partition noise. The ES laser might be more sensitive to optical feedback because there is a stronger modal competition as compared to the GS laser, which has a pretty flat optical spectrum around the central lasing wavelength. The latter could also be attributed to the ES degeneracy reflecting the existence of two closely spaced plevels leading to more additional radiative transitions. In order to better understand these observations, further 
experiments using lasers with the same cavity lengths will be performed, along with a study of distributed feedback (DFB) lasers under the same optical feedback configuration.

\section{CONCLUSIONS}

We have investigated for the first time the external optical feedback sensitivity of the GS and ES emission from two lasers made from the same QD material. Experimental results indicate that at the same bias level, the ES is more sensitive to external feedback than the GS. The ES laser can easily be perturbed by weak optical feedback, while the GS laser remains close to a free-running operation. Consequently, this study is of importance for the development of fiberpigtailed high-speed ES transmitters. Future work will compare ES and GS devices with the same cavity length as well as examine the quasi-periodic behavior observed in the ES laser, and its utilization under short external-cavity feedback.

\section{ACKNOWLEDGMENTS}

This work is supported by the Institut Mines-Télécom (IMT) through the Futur \& Ruptures program, by a public grant Nanodesign project funded by the IDEX Paris-Saclay, ANR-11-IDEX-0003-02 and by the DFG in the framework of SFB787.

\section{REFERENCES}

[1] O’Brien, D., Hegarty, S. P., Huyet, G., McInerney, J. G., Kettler, T., Laemmlin, M., Bimberg, D., Ustinov, V. M., Zhukov, A. E., Mikhrin, S. S., "Feedback sensitivity of $1.3 \mu \mathrm{m}$ InAs/GaAs quantum dot lasers," El. Lett. 39(25), 18191820 (2003).

[2] Azouigui, S., Dagens, B., Lelarge, F., Provost, J.-G., Make, D., Le Gouezigou, O., Accard, A., Martinez, A., Merghem, K., et al., "Optical Feedback Tolerance of Quantum-Dot- and Quantum-Dash-Based Semiconductor Lasers Operating at $1.55 \mu \mathrm{m}$," IEEE J. Sel. Top. Quantum Electron. 15(3), 764-773 (2009).

[3] Bimberg, D., "Quantum dot based nanophtonics and nanoelectronics" El. Lett. 44(3) 168-170 (2008)

[4] Bimberg, D., Kirstaedter, N., Ledentsov, N. N., Alferov, Z. I., Kop'ev, P. S., Ustinov, V. M., "InGaAs-GaAs quantum-dot lasers," IEEE J. Sel. Top. Quantum Electron. 3(2), 196-205 (1997).

[5] Grillot, F., Dagens, B., Provost, J., Su, H., Lester, L. F., "Gain Compression and Above-Threshold Linewidth Enhancement Factor in 1.3- $\mu \mathrm{m}$ InAs-GaAs Quantum-Dot Lasers," IEEE J. Quantum Electron. 44(10), 946-951 (2008). [6] Crowley, M. T., Naderi, N. A., Su, H., Grillot, F., Lester, L. F., "GaAs-Based Quantum Dot Lasers," Advances in Semiconductor Lasers, 371-417 (2012).

[7] Heitz, R., Guffarth, F., Mukhametzhanov, I., Grundmann, M., Madhukar, A., Bimberg, D., "Many-body effects on the optical spectra of InAs/GaAs quantum dots," Phys. Rev. B 62(24), 16881 (2000).

[8] Arsenijević, D., Schliwa, A., Schmeckebier, H., Stubenrauch, M., Spiegelberg, M., Bimberg, D., Mikhelashvili, V., Eisenstein, G., "Comparison of dynamic properties of ground- and excited-state emission in p-doped InAs/GaAs quantum-dot lasers,” Appl. Phys. Lett., 104(18), 181101 (2014)

[9] Xu, P.-F., Ji, H.-M., Xiao, J.-L., Gu, Y.-X., Huang, Y.-Z., Yang, T., "Reduced linewidth enhancement factor due to excited state transition of quantum dot lasers," Opt. Lett. 37(8), 1298 (2012).

[10] Zubov, F. I., Maximov, M. V., Moiseev, E. I., Savelyev, A. V., Shernyakov, Y. M., Livshits, D. A., Kryzhanovskaya, N. V., Zhukov, A. E., "Observation of zero linewidth enhancement factor at excited state band in quantum dot laser," Electron. Lett. 51(21), 1686-1688 (2015).

[12] Kovsh, A. R., Maleev, N. A., Zhukov, A. E., Mikhrin, S. S., Vasil'ev, A. P., Semenova, E. A., Shernyakov, Y. M., Maximov, M. V., Livshits, D. A., Ustinov, V. M., Ledentsov, N. N., Bimberg D., Alferov, Zh. I., "InAs/InGaAs/GaAs quantum dot lasers of $1.3 \mu \mathrm{m}$ range with enhanced optical gain," J. Cryst. Growth 251(1-4), 729-736 (2003).

[13] Otto, C., Lüdge, K., Schöll, E., "Modeling quantum dot lasers with optical feedback: sensitivity of bifurcation scenarios," Phys. Status Solidi B 247, 829-845 (2010).

[14] Stevens, B. J., Childs, D. T. D., Shahid, H., Hogg, R. A., "Direct modulation of excited state quantum dot lasers," Appl. Phys. Lett. 95(6), 061101 (2009).

[15] Lin, G., Tang, H., Cheng, H., Chen, H., "Analysis of Relative Intensity Noise Spectra for Uniformly and Chirpily Stacked InAs-InGaAs-GaAs Quantum Dot Lasers,” J. Light. Technol. 30(3), 331-336 (2012). 